\title{
Comparison of liver enzymes and sonological grading in nonalcoholic fatty liver
}

\author{
Bishnu Jwarchan', Subita Lalchan², Anil Dhakal ${ }^{3}$, Ramesh R Acharya ${ }^{1}$ \\ ${ }^{1}$ Lecturer, Department of Internal Medicine, Manipal Teaching Hospital, Pokhara, Nepal, ${ }^{2}$ Assistant Professor, \\ Department of Radio-Diagnosis, Manipal Teaching Hospital, Pokhara, Nepal, ${ }^{3}$ MD Resident, Department of Internal \\ Medicine, Manipal Teaching Hospital, Pokhara, Nepal
}

Background: Nonalcoholic fatty liver disease (NAFLD) is the leading cause of diffuse liver disease with a global prevalence of $25.24 \%$ and progresses to fibrosis and nonalcoholic steatohepatitis (NASH). Diagnosing NAFLD requires demonstration of increased liver fat and ultrasound imaging is widely used for screening. Specific blood tests to diagnose NAFLD and NASH are not yet available and alanine transaminase (ALT) has been used as a marker in population based studies. Aims and Objective: This study was aimed to compare the liver enzymes and sonological grading in NAFLD. Materials and Methods: A prospective hospital based observational study was carried out in 85 patients presenting to medical outpatient department (OPD) with ultrasonogical evidence of nonalcoholic fatty liver (NAFL) from January 2016 to December 2016. The patients were sent for liver enzymes and serum ALT, aspartate aminotransferase (AST) and gamma-glutamyltransferase (GGT) were particularly correlated with the grade of NAFL.Data were collected on a preformed proformaand analyzed using the Statistical Package for the Social Sciences (SPSS)20.0 (SPSS Inc., Chicago, IL, USA). Results: In our study, mean age of the patients was 46.08 years and most of the patients were in age group of $40-60$ years. Females $(65.88 \%)$ were more affected than males (34.12\%). There were $71(83.5 \%)$ patients with grade 1 NAFL and $14(16.5 \%)$ patients with grade 2 NAFL. The level of serum ALT increased with higher grades of NAFL. The mean ALT in grade 1 NAFL was 29.14 19.41 and in grade 2 NAFL was 42.19 2157 ; $p=0.027$. Comparison of serum AST and GGT with ultrasonological grading did not show statistical difference. Conclusion: Serum ALT levels correlated with the ultrasonological grades of NAFLD ( $p=0.027$ ) whereas AST and ALT did not show statistical correlation with grades of fatty liver. Our study suggests for large sample size study for AST and GGT values relationship with ultrasonological grades.

Key words: Fatty liver; Liver enzymes; Nonalcoholic fatty liver disease (NAFLD); Ultrasonography

\section{INTRODUCTION}

Nonalcoholic fatty liver disease (NAFLD) is defined as the presence of $\geq 5 \%$ of hepatic steatosis (HS), either by imaging or by histology, in the absence of secondary causes of hepatic fat accumulation, such as chronic viral hepatitis, medications that induce steatosis, and other chronic liver diseases,such as autoimmune hepatitis, hemochromatosis, Wilson's disease, or significant alcohol consumption. Ongoing or recent alcohol consumption $>21$ standard drinks on average per week in men and $>14$ standard drinks
http://nepjol.info/index.php/AJMS DOI: 10.3126/ajms.v11i2.27203 E-ISSN: 2091-0576 P-ISSN: 2467-9100 
progression to fibrosis and $0.09 \%$ mean annual rate of progression in NASH. ${ }^{2}$ Thus the estimation of hepatic fat accumulation is of utmost importance.

Diagnosing NAFLD requires demonstration of increased liver fat. Ultrasound imaging, which allows subjective estimation of fatty infiltration in the liver is widely used for screening but has a low performance for the detection of mild steatosis. To overcome this limitation, some semiquantitative scores have been proposed. ${ }^{4}$

Specific blood tests to diagnose NAFLD and NASH are not yet available. However, population-based studies that have used serum alanine transaminase (ALT) as a marker of liver injury indicate that study populations have ALT elevations that cannot be explained by excessive alcohol consumption, other known causes of fatty liver disease, viral hepatitis, or drug-induced or congenital liver diseases. Such elevations of ALT increase with body mass index (BMI) and it is presumed to be due to NASH. ${ }^{5}$ Our study was carried out to compare the pattern of liver enzymes with ultrasonological grading of fatty liver.

\section{MATERIALS AND METHODS}

A prospective hospital based observational study was carried out in patients with ultrasonological findings of nonalcoholic fatty liver (NAFL) presenting to medical OPD of Manipal Teaching Hospital in between $1^{\text {st January }} 2016$ to $30^{\text {th }}$ December 2016, with an objective to compare and correlate their sonological grading of NAFL with liver enzymes. A sample size of 85 such patients was taken and any patients found to have NAFL in their ultrasound report were sent for liver enzymes after a detailed clinical evaluation. Serum ALT, aspartate transaminase (AST) and gamma-glutamyl transferases (GGT) were particularly correlated with the grade of NAFL. Data were collected on a preformed proforma which included the patients age, sex, grade of NAFL and values of serum ALT, AST and GGT. An informed consent was taken from each patient included in this study. Data were analyzed using the SPSS software for Windows (version 20, SPSS Inc., Chicago, IL, USA). Data were expressed using descriptive statistics such as mean, standard deviation for continuous variables, frequency, and percentage for categorical variables. Statistical analysis was done using unpaired $t$ test for comparison of serum liver enzymes in between various grades of NAFL with 5\% significance level.

\section{RESULTS}

There were total 85 patients with NAFL presenting to medical OPD of Manipal Teaching Hospital in between $1^{\text {st }}$ January 2016 to $30^{\text {th }}$ Dec 2016. Mean age of the patients was 46.08 (SD 11.93) and ranged between 25 to 78 years of age. Most of the patients with NAFL were in the age group 40-60 years (Figure 1). Females $(65.88 \%)$ were more affected than males $(34.12 \%)$ (Figure 2). Ultrasound report suggested that there were $71(83.5 \%)$ patients with grade 1 NAFL and $14(16.5 \%)$ patients with grade 2 NAFL. We didn't have any case with grade 3 NAFL. The mean ALT in grade 1 NAFL was $29.14 \pm 19.41$ and in grade 2 NAFL was 42.19 \pm 21.57 . The mean AST in grade 1 NAFL was $26.20 \pm 17.47$ and in grade 2 was $33.44 \pm 19.25$. The mean GGT in grade 1 NAFL was $38.84 \pm 30.59$ and in grade 2 was $34.36 \pm 5.670$. Serum ALT was the only liver enzyme showing statistical significant difference in between grade 1 and grade 2 NAFL ( $p=0.027)$ suggesting the level of serum ALT increased significantly with higher grades of NAFL (Table 1).

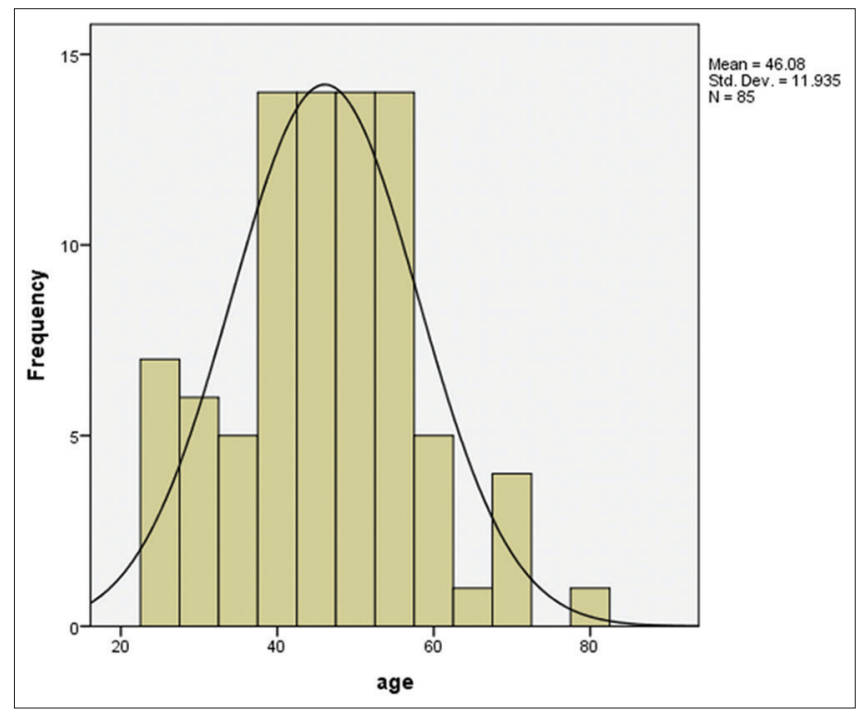

Figure 1: Histogram showing age distribution of patients with NAFL

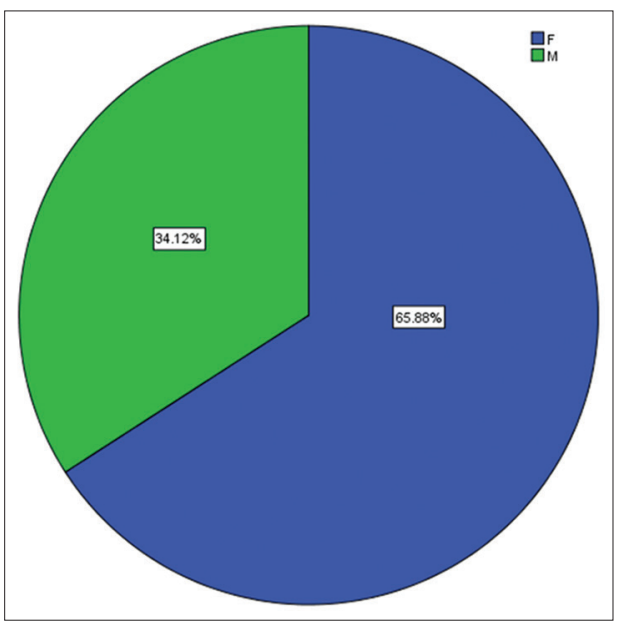

Figure 2: Pie chart showing sex distribution of patients with NAFL 


\begin{tabular}{|c|c|c|c|c|c|}
\hline Liver Enzymes & Grade1NAFLD (71) & Grade2 NAFLD (14) & Total (85) & $\mathrm{t}$ value & p \\
\hline $\begin{array}{l}\text { ALT } \\
(\text { mean/SD) }\end{array}$ & $\begin{array}{c}29.14 \\
(19.414)\end{array}$ & $\begin{array}{c}42.19 \\
(21.574)\end{array}$ & $\begin{array}{c}31.29 \\
(20.224)\end{array}$ & -2.257 & 0.027 \\
\hline $\begin{array}{l}\text { AST } \\
\text { (mean/SD) }\end{array}$ & $\begin{array}{c}26.20 \\
(17.479)\end{array}$ & $\begin{array}{c}33.44 \\
(19.257)\end{array}$ & $\begin{array}{c}27.39 \\
(17.869)\end{array}$ & -1.393 & 0.167 \\
\hline $\begin{array}{l}\text { GGT } \\
\text { (mean/SD) }\end{array}$ & $\begin{array}{c}38.84 \\
(30.591)\end{array}$ & $\begin{array}{l}34.36 \\
(5.670)\end{array}$ & $\begin{array}{c}38.10 \\
(28.033)\end{array}$ & 0.543 & 0.588 \\
\hline
\end{tabular}

\section{DISCUSSION}

The mean age of the patients in our study was 46.08 years and ranged between 25 to 78 years of age. Macabuag-Oliva et al reported ages ranging from 20 to 76 years with a mean age of 51.27 years. $^{6}$ In their study Mahale et al reported the mean age of 49.73 years. $^{7}$ The majority of the cases in our study were in the age group 40 to 60 years. Similar findings were also reported in their study by Khanal et al, where majority of patients belonged to age group of 50 $59(32.1 \%)$, followed by $40-49(28.8 \%){ }^{8}$ The prevalence of fatty liver increased in individuals with advancing age to maximum of 40-49, 50-59 years of age and then decreased with advancing age in the study by Nomura et al. ${ }^{9}$ As one's lifestyle and dietary adherence changes with advancing age the prevalence of NAFLD also increases.

We found that more females $(65.88 \%)$ were affected than males $(34.12 \%)$. Uppalapati et al also reported that females had high risk of developing fatty liver disease in their study. ${ }^{10}$ Male and female patients comprised $37.6 \%$ and $62.4 \%$ of the cases of fatty liver in the study by Mahale et al. ${ }^{7}$ However Singh et al reported the incidence of fatty liver to be more in males than in females. ${ }^{11}$

In our study most of the cases were grade 1 fatty liver. There were $71(83.5 \%)$ patients with grade 1 NAFL and $14(16.5 \%)$ patients with grade 2 NAFL. Khanal et al reported grade 1 fatty liver $(68.8 \%)$, followed by grade $2(28 \%)$ and $2.8 \%$ of grade 3 fatty liver. ${ }^{8}$ Similarly Mahaling et al reported that the prevalence of NAFLD grade 1 was $47.1 \%$, grade 2 was $42.8 \%$, and $10 \%$ had grade 3 fatty liver. ${ }^{12}$ Such difference can be attributed to random selection of study subjects.

We found that serum ALT was the only liver enzyme showing statistically significant difference in between grade 1 and grade 2 NAFL ( $\mathrm{p}=0.027$ ) suggesting the level of serum ALT increased significantly with higher grades of NAFL. Dixon et al found that a raised index of insulin resistance, hypertension and raised alanine aminotransferase were independent predictors of NASH. ${ }^{13}$

In their study Macabuag-Oliva et al found that ALT level greater than $35 \mathrm{U} / \mathrm{L}$ has a sensitivity of $65 \%$ for detecting fatty liver and fibrosis in patients with a high probability and $56 \%$ for patients with moderate probability with a specificity of $45.95 \%$. But Ultrasound, ALT and AST showed no correlation with fibrosis score. ${ }^{6}$ High serum ALT and AST levels were seen in $13.8 \%$ and $35.5 \%$ cases of NAFLD respectively in their study by Mahale et al. ${ }^{7}$ However these results were not statistically significant.

Nevertheless, ALT has been used as a biomarker for various scoring systems used in the non-invasive diagnosis of NAFLD and NASH.

Also, in our study we included cases from ultrasonological diagnosis but biopsy was not done for the confirmation of NAFLD which is the major drawback. In addition, various scoring systems were also not used to correlate our findings.

\section{CONCLUSION}

Serum ALT levels correlated with the ultrasonological grades to diagnose NAFLD. We suggest large sample size studies for AST and GGT values relationship with ultrasonological grades.

\section{ACKNOWLEDGEMENT}

None.

\section{REFERENCES}

1. Chalasani N, Younossi Z, Lavine JE, Diehl MA, Brunt EM, Cusi K, et al. The diagnosis and management of non-alcoholic fatty liver disease: practice guideline by the American Association for the Study of Liver Diseases, American College of Gastroenterology, and the American Gastroenterological Association. Hepatology 2012; 55:2005-2023.

2. Younossi ZM, Koenig AB, Abdelatif D, Fasel $Y$, Henry $L$ and Wymer M. Global epidemiology of non-alcoholic fatty liver disease-meta-analytic assessment of prevalence, incidence and outcomes. Hepatology 2015; doi:10.1002/hep.28431

3. Chalasani N, Younossi Z, Lavine JE,Chalrton M, Cusi K, Rinella M, et al. The Diagnosis and Management of Nonalcoholic Fatty Liver Disease: Practice Guidance From the American Association for the Study of Liver Diseases. Hepatology 2018; 67(1):328-357.

4. Ferraioli $G$ and Soares Monteiro LB. Ultrasound-based techniques for the diagnosis of liver steatosis. World $\mathrm{J}$ 
Gastroenterol 2019; 25(40): 6053-6062.

5. Abdelmalek MF and Diehl AM. Nonalcoholic Fatty Liver Diseases and Nonalcoholic Steatohepatitis. In: Kasper DL, Fauci AS, Hauser SL, Longo DL, Jameson JL, Loscalzo J, eds. Harrison's principles of internal medicine. $20^{\text {th }}$ ed. New York: Mc Graw Hill, 2018, p 2401.

6. Macabuag-Oliva AM, Capellan ML and Benitez B. A comparison of the sensitivity and specificity of ultrasound elastography compared to liver ultrasound, ALT, and AST in the detection of fatty liver and fibrosis in patients with metabolic syndrome and type 2 diabetes mellitus. Journal of the ASEAN Federation of Endocrine Societies 2014;29(1):59.

7. Mahale AR, Prabhu SD, Nachiappan M, Fernandes M and Ullal S. Clinical relevance of reporting fatty liver on ultrasound in asymptomatic patients during routine health checkups. Journal of International Medical Research 2018; 46(11):4447-4454.

8. Khanal UP, Paudel B, Gurung G, Hu YS and Kuo CW Correlational study of nonalcoholic fatty liver disease diagnosed by ultrasonography with lipid profile and Body Mass Index in adult Nepalese population. J Med Ultrasound 2019; 27:19-25.

9. Nomura H, Kashiwagi S, Hayashi J, Kajiyama W, Tani S, Goto M, et al. Prevalence of fatty liver in a general population of Okinawa, Japan. Jpn J Med 1988; 27:142-149.

10. Uppalapati GP and Harish K. A study of clinical, biochemical and sonological profile of non-alcoholic fatty liver disease in type 2 diabetes patients. J Evid Based Med Healthc 2017; 4(90), 5414-5417.

11. Singh SP, Nayak S, Swain M, Rout N, Mallik RN, Agrawal O, et al. Prevalence of nonalcoholic fatty liver disease in coastal Eastern India: A preliminary ultrasonographic survey. Trop Gastroenterol 2004;25:76-79.

12. Mahaling DU, Basavaraj MM and Bika AJ. Comparison of lipid profile in different grades of nonalcoholic fatty liver disease diagnosed on ultrasound. Asian Pac J Trop Biomed 2013; 3:907-912.

13. Dixon JB, Bhathal PS and O'Brien PE. Nonalcoholic fatty liver disease: Predictors of nonalcoholic steatohepatitis and liver fibrosis in the severely obese. Gastroenterology 2001; 121:91-100.

\footnotetext{
Authors Contribution: RRA-Concept of study, manuscript preparation and review of study

Work attributed to:

Department of Internal Medicine, Manipal Teaching Hospital

Orcid ID:

Dr. Bishnu Jwarchan - (D http://orcid.org/0000-0001-5190-5975

Dr. Subita Lalchan - (1) http://orcid.org/0000-0001-6509-6304

Dr. Anil Dhakal - (i) http://orcid.org/0000-0002-3486-1357

Dr. Ramesh R Acharya - ittp://orcid.org/0000-0002-0930-9878

Source of funding: None, Conflict of interest: None.
}

BJ-Concept and design of the study, reviewed the literature, manuscript preparation and critical revision of the manuscript; SL- Concept, collected data, statistically analyzed and interpreted and helped in preparing first draft of manuscript; AD- Collected data, literature search, prepared first draft of manuscript; 